OPEN ACCESS

Edited by:

Aurel Popa-Wagner,

University of Rostock, Germany

Reviewed by:

Umesh Gangishetti,

Emory University, United States

Wei Wang,

Stowers Institute for Medical

Research, United States

${ }^{*}$ Correspondence:

Chiung-Chih Chang

neur099@adm.cgmh.org.tw

Received: 19 May 2017 Accepted: 05 December 2017

Published: 10 January 2018

Citation:

Chang Y-T, LU C-H, WU M-K, Hsu S-W, Huang C-W, Chang W-N,

Lien C-Y, Lee J-J and Chang C-C (2018) Salience Network and Depressive Severities in Parkinson's Disease with Mild Cognitive Impairment: A Structural

Covariance Network Analysis.

Front. Aging Neurosci. 9:417. doi: 10.3389/fnagi.2017.00417

\section{Salience Network and Depressive Severities in Parkinson's Disease with Mild Cognitive Impairment: A Structural Covariance Network Analysis}

\author{
Ya-Ting Chang', Cheng-Hsien Lu', Ming-Kung Wu'2, Shih-Wei Hsu ${ }^{3}$, Chi-Wei Huang ${ }^{1}$, \\ Wen-Neng Chang ${ }^{1}$, Chia-Yi Lien ${ }^{1}$, Jun-Jun Lee ${ }^{1}$ and Chiung-Chih Chang ${ }^{1 *}$ \\ ${ }^{1}$ Department of Neurology, Kaohsiung Chang Gung Memorial Hospital, Chang Gung University College of Medicine, \\ Kaohsiung, Taiwan, ${ }^{2}$ Department of Psychiatry, Kaohsiung Chang Gung Memorial Hospital, Chang Gung University College \\ of Medicine, Kaohsiung, Taiwan, ${ }^{3}$ Department of Radiology, Kaohsiung Chang Gung Memorial Hospital, Chang Gung \\ University College of Medicine, Kaohsiung, Taiwan
}

Purpose: In Parkinson's disease with mild cognitive impairment (PD-MCl), we investigated the clinical significance of salience network $(\mathrm{SN})$ in depression and cognitive performance.

Methods: Seventy seven PD-MCl patients that fulfilled multi-domain and non-amnestic subtype were included. Gray matter structural covariance networks were constructed by 3D T1-magnetic resonance imaging and seed based analysis. The patients were divided into two groups by psychiatric interviews and screening of Geriatric Depression Scale (GDS): PD-MCI with depression (PD-MCI-D) or without depression (PD-MCl-ND). The seed or peak cluster volume, or the significant differences in the regression slopes in each seed-peak cluster correlation, were used to evaluate the significance with the neurobehavioral scores.

Results: This study is the first to demonstrate that the PD-MCI-ND group presented a larger number of voxels of structural covariance in SN than the PD-MCI-D group. The right fronto-insular seed volumes and the peak cluster of left lingual gyrus showed significant inverse correlation with the Geriatric Depression Scale (GDS; $r=-0.231$, $P=0.046)$.

Conclusions: This study is the first to validate the clinical significance of the SN in PD$\mathrm{MCl}-\mathrm{D}$. The right insular seed value and the SN correlated with the severity of depression in $\mathrm{PD}-\mathrm{MCl}$

Keywords: brain imaging, cognition, depression, mood disorders, neuroimaging

\section{INTRODUCTION}

Although Parkinson's disease with mild cognitive impairment (PD-MCI) is conceptualized as a transitional state between normal cognition and dementia, it is a major predictor for the conversion of dementia (Domellof et al., 2015). The quality of life in patients with PD-MCI is limited (Reginold et al., 2013), and the presence of depression is one of the most common non-motor symptoms 
(Burn, 2002; Aarsland et al., 2012). PD-MCI with depression (PDMCI-D) has also been identified as a risk factor for dementia (Modrego and Ferrandez, 2004; Janvin et al., 2006), and the use of cholinesterase inhibitors has been reported to delay the cognitive progression (Lu et al., 2009). For PD-MCI, the natural history of PD-MCI-D does not parallel that of motor symptoms, suggesting the distinctive pathophysiological mechanism (Brown and Jahanshahi, 1995). In this context, it is of clinical relevance to explore the neural basis of PD-MCI-D.

In Parkinson's disease, the salience network (SN) has been implicated in emotional processing and depression (Remy et al., 2005). Several limbic structures were reported in SN that included the insula and anterior cingulate cortex (ACC) (Seeley et al., 2007; Bressler and Menon, 2010). The reduced Blood oxygenation level dependent (BOLD) response and reduced gray matter (GM) volume within the SN in Parkinson's disease have been reported to mediate the emotional valence (Cardoso et al., 2009; Kostic et al., 2010). In PD-MCI, the GM atrophy of SN (Beyer et al., 2007; Song et al., 2011) showed mixed evidence for predicting the conversion to dementia (Aybek et al., 2009; Jokinen et al., 2009; Martin et al., 2009). Despite the solid evidence of SN in PD with depression (Beyer et al., 2007; Kostic et al., 2010; Song et al., 2011), whether the changes in the $\mathrm{SN}$ also mediate the depression state in PD-MCI is not fully investigated.

The current neuronal scaffold of neurodegenerative diseases emphasizes the pathological protein accumulation within the large-scale networks that are anatomically distinctive (Seeley et al., 2009). The application of structural covariance networks (SCNs) has been supported by recent research in that highly related regions may show covariance in morphometric characteristics. SCN patterns have been shown to be associated with structural or functional connectivity while the structural covariance strength often reflects how close two interconnected hubs interact (Alexander-Bloch et al., 2013). The SCN of SN included the insular seed and peak clusters in the prefrontal cortex, ACC, left angular gyrus, and medial and lateral temporal cortex (Clos et al., 2014). The covariance strength between seed and peak clusters has been found to associated with social cognition, reward, explicit memory, and negative emotion (Zaki et al., 2007; Jabbi and Keysers, 2008; Clos et al., 2014). The SCN might serve as a potential model for understanding whether the $\mathrm{SN}$ was involved in depressive state or severity in PD-MCI.

To date, there is no literature for depressive network analysis in PD-MCI. This study explored whether the SN may signify PDMCI-D. Meanwhile, we explored whether the seed or peak cluster volume or the seed-peak covariance strength may determine the cognitive performance or depressive scores in patients with PD-MCI.

\section{MATERIALS AND METHODS}

\section{Subjects}

The study patients were treated at the Department of Neurology, Kaohsiung Chang Gung Memorial Hospital. This study was approved by the Chang Gung Memorial Hospital Ethics Committee. A total of 89 subjects with PD-MCI (48 male and 41 female subjects) were included after the consensus of a panel composed of neurologists, neuropsychologists, neuroradiologists, psychiatrists, and experts in nuclear medicine (Huang et al., 2015). PD-MCI is diagnosed according to Movement Disorder Society Task Force criteria (Litvan et al., 2012) and the result of formal neuropsychological testing (Chang et al., 2008, 2009) that the cognitive impairment is not sufficient to interfere with functional independence (Litvan et al., 2012). As there were clinical heterogeneities among patients with PD-MCI, only those with multiple domains non-amnestic presentations were included $(n=77)$. The diagnosis of depression was carried out by means of a half-hour structured interview by a psychiatrist according to the Diagnostic and Statistical Manual of Mental Disorders, Fourth Edition (DSM-IV) criteria (Zimmerman et al., 2011). A total of 35 patients were in the PD-MCI-D group, and 42 were in the PD-MCI without depression (PD-MCI-ND) group. All participants were matched for age and years of education and patients were matched for disease severity as measured by the Unified Parkinson Disease Rating Scale III (UPDRS-III) and Hoehn and Yahr scale. A voluntary comparison normal control (NC) group, who had no underlying neurological or psychiatric disorders, was recruited from outpatient neurological and geriatrics clinics.

\section{Clinical and Neurobehavioral Assessments}

We used a comprehensive battery of tests to assess the cognitive ability of all participants. The Mini-Mental State Examination (MMSE) assesses the general intellectual function. The episodic memory was assessed by the Chinese version verbal learning test (CVVLT) using a 9-word list with fixed order over 4 learning trials (Chang et al., 2016). The scores after a 30-s (CVVLT$30 \mathrm{~s}$ ) and 10 -min delay (CVVLT-10 $\mathrm{m}$ ) were recorded. The semantic verbal fluency tests included the free generations of four categories (animal, fruit, town, and transportation), each for $1 \mathrm{~min}$. The Visual Object and Space Perception Battery (VOSP) and the copy of modified Rey-Osterrieth complex figure and pentagons were used to assess the visual-spatial abilities (Rapport et al., 1998). The subjects' frontal lobe function was assessed using digit-forward, digit-backward, Stroop interference (Amieva et al., 2004), and modified Trails B tests (Reitan, 1955).

All the patients with PD-MCI completed the Chinese version of the 15-item Geriatric Depression Scales (GDS-15) (Spreen and Strauss, 1998). For illiterate subjects, the scale was read by the interviewers without any comment, and the subjects were asked to choose one of the answers. All patients with PD-MCID, previously confirmed by the psychiatrist, showed a higher GDS screening score (Table 1) and all were higher than the cutoff value (Marc et al., 2008).

\section{Magnetic Resonance Imaging (MRI) Acquisition}

The T1-weighted sequence, inversion-recovery-prepared, threedimensional, spoiled, gradient-recalled acquisition in steadystate sequences were acquired using the GE 3T Signa Excite scanner (GE Medical System, Milwaukee, WI, United States) 
with the following parameters: repetition time/inversion time of $8,600 \mathrm{~ms} / 450 \mathrm{~ms}, 240 \mathrm{~mm} \times 240 \mathrm{~mm}$ field of view, and 1-mm slice thickness.

\section{MRI Preprocessing}

Structural images were preprocessed using voxel-based morphometry (VBM) implemented with Statistical Parametric Mapping 8 software $^{1}$ running under MATLAB 7.9 (MathWorks, Natick, MA, United States). VBM is a whole-brain, unbiased, and semi-automated technique. First, structural images were normalized to the Montreal Neurological Institute stereotactic space and then segmented to extract the GM. Using diffeomorphic anatomical registration via the exponentiated lie algebra approach, related tissue segments were used to create a custom template. The resulting GM images were finally smoothed with an 8-mm isotropic Gaussian kernel (Mechelli et al., 2004).

\section{Statistical Analysis}

Voxel-based morphometry was used to investigate the atrophic patterns among the three clinical groups with specific $\mathrm{T}$ contrasts as follows: NC > PD-MCI-ND, NC > PD-MCI-D, PD-MCI$\mathrm{D}>\mathrm{PD}-\mathrm{MCI}-\mathrm{ND}$ or PD-MCI-D < PD-MCI-ND. The threshold for the $t$-test parametric maps was corrected for multiple

${ }^{1}$ http://www.fil.ion.ucl.ac.uk/spm/ comparisons using the family-wise error (FWE) correction and with the significance threshold at $P<0.001$ and cluster size $>100$ voxels.

We used seed-based analysis (Lin et al., 2016) to construct the SCN. Regional densities were extracted from the 4-mm radius sphere of the right fronto-insula seed $(x=36, y=18$, $z=4$ ) (Seeley et al., 2007; Menon, 2015) and used to model the regional densities in all voxels of the preprocessed GM segments. The PD-MCI-D and PD-MCI-ND groups were separately modeled. For each group, the specific contrast was set to identify voxels (peak clusters) that showed significant positive correlations between the right insular seed, represented physiologically as "structural associations" (Montembeault et al., 2016). Significant clusters were selected with Family-Wise Error rate (FWE) correction in Bonferroni method and a $P$-value less than 0.001 . Only clusters with more than 100 voxels were chosen.

For the group interaction test between the PD-MCI-D and PD-MCI-ND groups in the SN structural associations, statistical contrasts were set to identify seed-peak cluster voxels that expressed differences in the regression slopes. For the difference in the structural association, $\mathrm{T}$ contrasts were established to map the voxels that expressed stronger structural associations in the PD-MCI-ND group. The threshold for the resulting statistical parametric maps was established as FWE-corrected for multiple comparisons at $P<0.05$. Furthermore, voxels showing significant

TABLE 1 | Demographic data of patients with PD-MCl and normal controls.

\begin{tabular}{|c|c|c|c|c|}
\hline & Normal controls & PD-MCI & PD-MCI-ND & PD-MCI-D \\
\hline Age (year-old) & $63.4 \pm 8.1$ & $64.4 \pm 10.8$ & $65.8 \pm 9.8$ & $62.7 \pm 11.8$ \\
\hline Hoehn and Yahr scale & - & & $1.8 \pm 0.9$ & $1.6 \pm 0.7$ \\
\hline UPDRS & - & & $30.8 \pm 20.0$ & $27.1 \pm 14.7$ \\
\hline MMSE & $27.9 \pm 2.5$ & $25.9 \pm 3.8^{\mathrm{a}}$ & $25.9 \pm 3.7^{a}$ & $25.8 \pm 3.9^{a}$ \\
\hline CWVLT-30 s & $7.3 \pm 1.5$ & $6.52 \pm 1.8$ & $6.6 \pm 1.7$ & $6.5 \pm 2.1$ \\
\hline CWLT-10 min & $6.7 \pm 1.8$ & $6.1 \pm 2.0$ & $6.3 \pm 2.0$ & $5.9 \pm 1.9$ \\
\hline \multicolumn{5}{|l|}{ Visuospatial function } \\
\hline Modified R-O copy & $16.5 \pm 2.2$ & $16.0 \pm 2.5$ & $15.8 \pm 3.0$ & $16.3 \pm 1.7$ \\
\hline VOSP & $7.9 \pm 2.7$ & $7.4 \pm 2.8$ & $7.3 \pm 1.1$ & $7.1 \pm 1.6$ \\
\hline \multicolumn{5}{|l|}{ Frontal lobe function } \\
\hline Trail making test & $12.3 \pm 3.4$ & $10.6 \pm 4.4$ & $11.4 \pm 4.1$ & $9.7 \pm 4.6^{a}$ \\
\hline \multicolumn{5}{|c|}{ Semantic verbal fluency test } \\
\hline Animal & $15.7 \pm 4.9$ & $13.5 \pm 4.6^{\mathrm{a}}$ & $14.2 \pm 4.8$ & $12.7 \pm 4.3^{\mathrm{a}}$ \\
\hline Fruit & $12.7 \pm 3.4$ & $11.3 \pm 4.6$ & $11.2 \pm 2.6^{\mathrm{a}}$ & $11.4 \pm 6.3$ \\
\hline Town & $16.0 \pm 7.2$ & $11.7 \pm 6.3^{\mathrm{a}}$ & $11.4 \pm 6.1^{\mathrm{a}}$ & $12.1 \pm 6.7^{\mathrm{a}}$ \\
\hline Transportation & $9.5 \pm 3.2$ & $7.6 \pm 2.7^{a}$ & $7.8 \pm 2.3^{a}$ & $7.3 \pm 3.2^{\mathrm{a}}$ \\
\hline GDS & $2.9 \pm 3.2$ & $5.1 \pm 3.9^{a}$ & $2.1 \pm 1.3$ & $8.6 \pm 3.0^{\mathrm{ab}}$ \\
\hline
\end{tabular}

a $P<0.05$, compared to normal controls. ${ }^{b} P<0.05$, compared to PD-MCI-ND. CWLT, Chinese version of the Verbal Learning Test; GDS, Geriatric Depression Scale; MMSE, Mini-Mental State Examination; PD-MCl, Parkinson's Disease with mild cognitive impairment; PD-MCI-ND, PD-MCl without depression; PD-MCI-D, PD-MCl with depression; R-O, Rey-Osterrieth; UPDRS, Unified Parkinson's Disease Rating Scale; VOSP, Visual Object and Space Perception Battery. 
differences in the regression slopes in each seed-peak cluster correlation were compared. Then, a $4-\mathrm{mm}$ radius sphere was placed on these peak voxels, and the GM volume was used to evaluate the clinical significance of these regions. Neurocognitive test scores were the dependent variable in the linear regression model with the seed or peak voxel volume as the predictor, and possible covariates such as GDS scores, age, and education were adjusted. GDS scores were also a dependent variable in the linear regression model with the seed or peak voxel volume as the predictor, and covariates of age and education were adjusted.

Clinical and laboratory data were expressed as mean \pm standard deviation. Analysis of variance with Bonferroni correction for multiple comparisons was used to compare continuous variables among the NC, PD-MCI-D and PDMCI-ND. All statistical analyses were conducted using SPSS software (SPSS version 22 for Windows ${ }^{\circledR}$, SPSS Inc., Chicago, IL, United States). Statistical significance was set at $p<0.05$.

\section{RESULTS}

\section{Demographic and Clinical Characteristics}

Seventy seven patients with PD-MCI (35 with PD-MCI-D and 42 with PD-MCI-ND) and $27 \mathrm{NC}$ matched for age, gender, and educational levels completed the study (Table 1). The PD-MCI was lower than the NC in MMSE scores $(p=0.003)$, scores in digital forward and backward, lower semantic verbal fluency scores and higher GDS than NC $(P<0.05)$. Meanwhile, the PDMCI-D subgroup showed significantly lower scores in the frontal lobe function and semantic verbal fluency tests than NC group $(P<0.05)$. Except for the GDS scores, the comparisons of PDMCI-D and PD-MCI-ND groups, however, were not significant $(P>0.05)$.

\section{Brain Volume Atrophic Patterns}

Compared with controls, both the PD-MCI-ND and PD-MCI-ND (Figure 1A) groups showed atrophy in the bilateral temporal, frontal, and occipital lobes, and the right cerebellum. The right superior occipital and frontal gyri and the left supramarginal gyrus were highlighted for the difference in regional brain volume observed in the PD-MCI-D and PD-MCI-ND groups when compared with the NC group $(p<0.001)$. The differences in the brain volumes between the PD-MCI-ND and PD-MCI-D groups were observed in the right fusiform gyrus, right hippocampus, and bilateral superior parietal gyri $(p<0.001$; Figures 1B,C). The details of the brain areas with significant volumetric differences are listed in Table 2.

The relationship between more atrophic clusters in the PDMCI-ND group with the selected cognitive test was further explored. After controlling for age and education, the GDS was positively correlated with volume in the right fusiform gyrus $(r=0.351, P=0.002)$ and the left superior parietal lobe $(r=0.368, P=0.001)$. The VOSP was positively correlated with volume in right hippocampus $(r=0.305, P=0.008)$ and the right superior parietal lobe $(r=0.332, P=0.004)$.

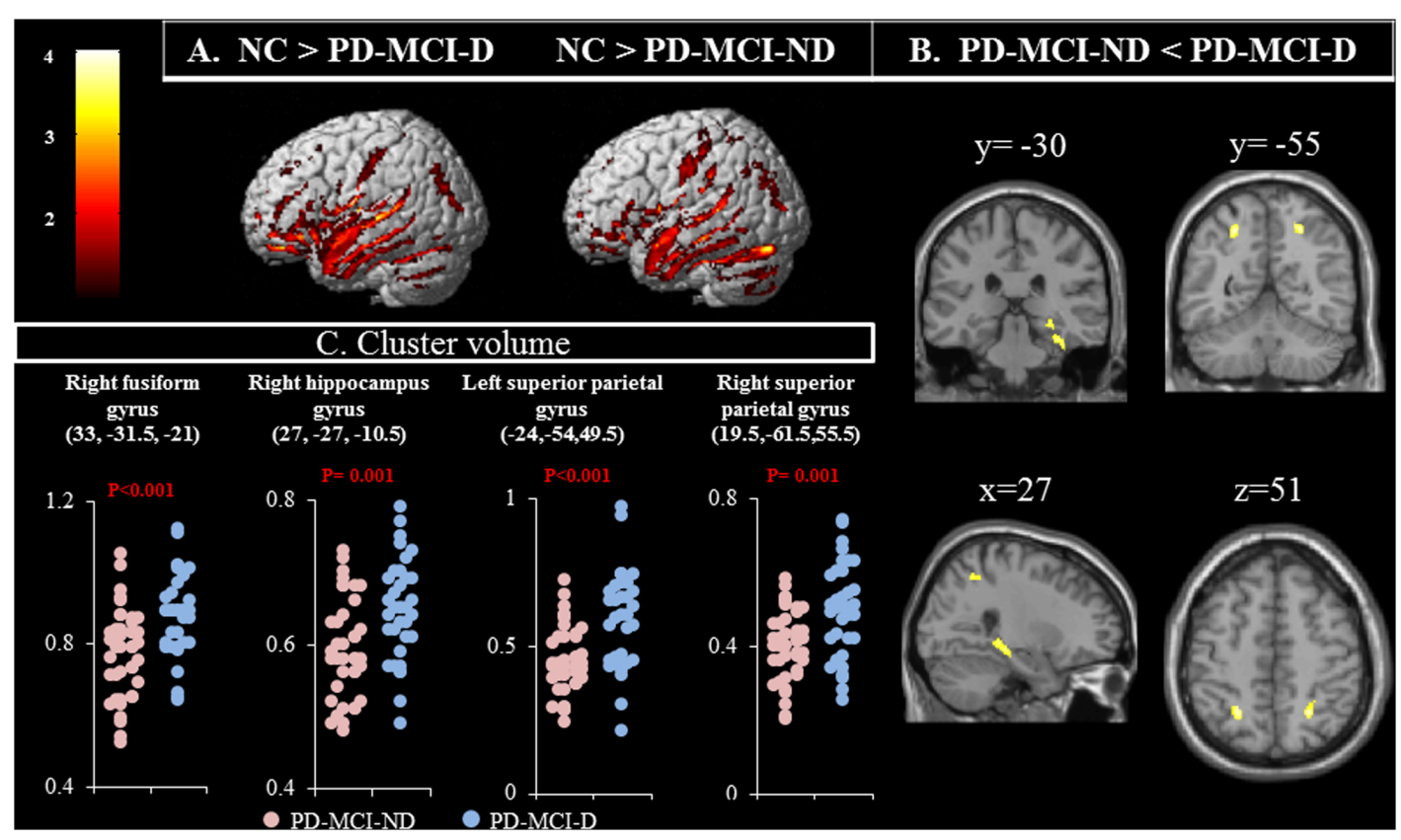

FIGURE 1 | (A) Contrast maps of volume changes depicting the brain areas with a significant difference in the gray matter volume in normal controls (NC) vs. patients with Parkinson's disease-mild cognitive impairment without depression (PD-MCl-ND) and vs. PD-MCI with depression (PD-MCl-D), using voxel-based morphometric methods. (B) Contrast maps between PD-MCl-ND and PD-MCI-D. Z-statistic maps $[P<0.001$, corrected for a family-wise error (FWE) and extended cluster voxels > 100]. (C) Plot of significant difference between PD-MCI-D and PD-MCI-ND in cluster volume. 


\section{Patterns of Structural Associations in the PD-MCI-ND and PD-MCI-D Groups}

The representative right insular seed is shown in Figure 2A, and the seed volume was significantly higher in the PD-MCIND group (Figure 2B). Using the seed and correlation analysis, the spatial patterns of SN in the PD-MCI-ND (Figure 2C) and PD-MCI-D (Figure 2D) groups share similar patterns (Table 3). However, the PD-MCI-ND group presented a larger number of voxels (the insula, hippocampus, and ACC: 44934 voxels) than PD-MCI-D (the insula, hippocampus, and ACC: 6421 voxels).

\section{Covariance Strength Interactions between the Two PD-MCI Groups}

There were eight clusters that showed covariance strength interactions between the two PD-MCI groups (Figures $\mathbf{3 A - H}$ ), all showing stronger covariance coefficients in the PD-MCI-ND group. These included the left ACC, left calcarine, left caudate, right fronto-insula cortex, right hippocampus, right inferior frontal gyrus, left lingual gyrus volume, left rolandic operculum, and right superior frontal lobe (Figure 3).

\section{Seed or Peak Cluster Volumes and Relationships with Neurobehavioral Scores}

The peak clusters showing differences in the brain volumes between the PD-MCI-ND and PD-MCI-D groups (Figure 4A) were positive correlated with GDS in right fusiform gyrus and left superior parietal gyrus, and positive correlated with VOSP in right hippocampus and right superior parietal gyrus $(p<0.05)$ (Figure 4B). Other neurobehavioral scores did not correlate with the peak clusters volume $(P>0.05)$.

For SN seed and peak clusters, we first explored whether the seed region volume was correlated with the selected cognitive test. Since age showed inverse correlation with GDS ( $r=-0.249, P=0.029)$, verbal fluency scores in the fruit category $(r=-0.294, P=0.009)$ and VOSP scores $(r=-0.367$, $P=0.001)$ correlation was analyzed after controlling for age.

TABLE 2 | Voxel-based morphometry (VBM) difference among normal controls and patients with PD-MCI-ND and PD-MCI-D.

\begin{tabular}{|c|c|c|c|c|c|}
\hline & $x$ & $y$ & $z$ & $T$-score & Voxels \\
\hline \multicolumn{6}{|l|}{ NC > PD-MCl-ND } \\
\hline Right temporal lobe & 37.5 & -13.5 & -12 & 29.1729 & 25398 \\
\hline Right cerebellum & 30 & -27 & -31.5 & 15.0927 & 7230 \\
\hline Left frontal lobe & -21 & 48 & -21 & 9.7238 & 120 \\
\hline Right middle temporal gyrus & 58.5 & -6 & -15 & 7.4086 & 146 \\
\hline Left middle occipital gyrus & -34.5 & -73.5 & 25.5 & 12.7509 & 268 \\
\hline Right calcarine gyrus & 16.5 & -70.5 & 15 & 11.2434 & 166 \\
\hline Right middle occipital gyrus & 37.5 & -67.5 & 31.5 & 12.0893 & 253 \\
\hline Right superior occipital gyrus & 22.5 & -64.5 & 31.5 & 11.8185 & 169 \\
\hline Left supramarginal gyrus & -49.5 & -34.5 & 33 & 13.149 & 100 \\
\hline Right supramarginal gyrus & 51 & -30 & 36 & 13.0843 & 135 \\
\hline Left middle frontal gyrus & -24 & 31.5 & 42 & 9.1489 & 143 \\
\hline Right middle frontal gyrus & 25.5 & 19.5 & 52.5 & 7.6016 & 142 \\
\hline \multicolumn{6}{|l|}{ NC > PD-MCI-D } \\
\hline Right temporal lobe & 37.5 & -13.5 & -12 & 28.6554 & 25033 \\
\hline Right cerebellum & 30 & -27 & -31.5 & 14.794 & 6511 \\
\hline Left frontal lobe & -21 & 48 & -21 & 9.4818 & 121 \\
\hline Right middle temporal gyrus & 58.5 & -6 & -15 & 7.2187 & 127 \\
\hline Right middle frontal gyrus & 34.5 & 40.5 & 15 & 7.4607 & 100 \\
\hline Left middle occipital gyrus & -34.5 & -73.5 & 25.5 & 12.4705 & 247 \\
\hline Right calcarine gyrus & 16.5 & -70.5 & 15 & 11.0682 & 163 \\
\hline Right middle occipital gyrus & 37.5 & -67.5 & 31.5 & 11.8221 & 217 \\
\hline Right superior occipital gyrus & 22.5 & -64.5 & 31.5 & 11.5668 & 163 \\
\hline Right supramarginal gyrus & 51 & -30 & 36 & 12.7912 & 131 \\
\hline Left middle frontal gyrus & -24 & 31.5 & 42 & 8.9764 & 141 \\
\hline Right superior frontal gyrus & 25.5 & 21 & 51 & 7.502 & 142 \\
\hline \multicolumn{6}{|l|}{ PD-MCI-D > PD-MCI-ND } \\
\hline Right fusiform gyrus & 33 & -31.5 & -21 & 3.9212 & 103 \\
\hline Right hippocampus gyrus & 27 & -27 & -10.5 & 3.7796 & 108 \\
\hline Left superior parietal gyrus & -24 & -54 & 49.5 & 4.3339 & 122 \\
\hline Right superior parietal gyrus & 19.5 & -61.5 & 55.5 & 4.6819 & 138 \\
\hline
\end{tabular}

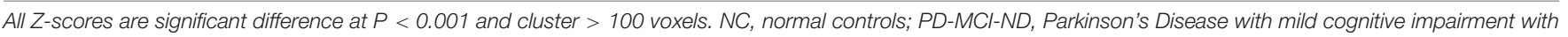
depression; PD-MCI-ND, Parkinson's Disease with mild cognitive impairment without depression; VBM, voxel-based morphometry. 


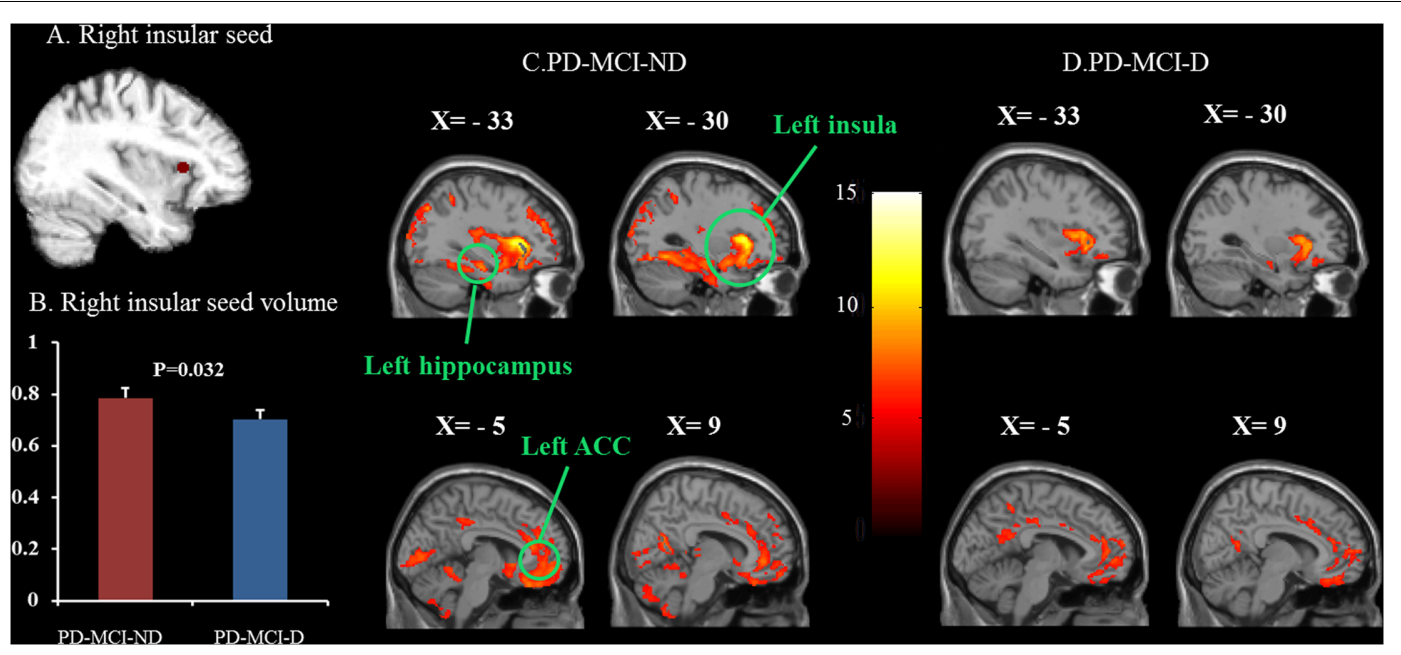

FIGURE 2 | Statistical maps depicting brain areas in which the gray matter intensity covaried with the frontoinsular seed (A), seed volume (B), and structural covariance networks in the PD-MCl-ND and PD-MCl-D groups (C,D). There was significant decrease in the seed volume in the PD-MCI-D group compared with the PD-MCl-ND group $(P=0.032)$. Z-statistic maps $(P<0.001$, corrected with an FWE and extended cluster voxels $>100)$. The images are displayed on a standard brain render.

After adequately controlling the possible covariate, the right fronto-insula seed (Figure 4C) volumes showed significant inverse correlation with the GDS $(r=-0.231, P=0.046)$ (Figure 4D). For the peak clusters showing differences in covariance strength between the $2 \mathrm{PD}$-MCI groups (Figure 4C), the left lingual gyrus volume was inversely correlated with the GDS ( $r=-0.266, P=0.021)$, the right hippocampal volume was significantly correlated with the verbal fluency scores in the fruit category $(r=0.270, P=0.010)$, and the left caudate volume was significantly correlated with VOSP scores $(r=0.233$, $P=0.046$ ) (Figure 4D). Other clusters volume did not correlate with neurobehavioral scores $(P>0.05)$.

\section{DISCUSSION}

\section{Main Findings}

By constructing the SCN, this study provides data on the influence of SN on depressive state and severity in PD-MCI. There are three major findings. First, smaller insular seed volumes were found in the PD-MCI-D group that also correlated with the GDS. Stronger seed-peak covariance strength was observed in the PD-MCI-ND group emphasizing the importance of SN. Second, the regression model suggests that the seed or peak cluster volume depicts specific cognitive scores, especially in the insula, left lingual gyrus, left caudate, and hippocampus. Lastly, the VBM analysis reveals the differences and similarities of atrophic patterns in PD-MCI-ND and PD-MCI-D groups. However, increased atrophy in the PD-MCI-ND group and structure-neurobehavioral correlation with GDS and VOSP cognitive test suggest compensatory mechanism in PD-MCID. This study forges a link between SN and PD-MCI-D and also validates the data by previous PD-MCI studies (Beyer et al., 2007; Aybek et al., 2009; Jokinen et al., 2009; Martin et al., 2009; Song et al., 2011) between SN and neurobehavioral function.

\section{The Role of Insular Seed in PD-MCI-D}

Lower insular seed was found in our PD-MCI-D patients, compared with the PD-MCI-ND. According to Braak's staging hypothesis of PD (Braak et al., 2006), the insula is one of the vulnerable regions by alpha-synuclein deposition. Changes in the activity and volume of insula in depression suggest a critical role of this region in modulating emotional perception. Depression in patients with PD is found to correlate with reduced serotonin $1 \mathrm{~A}$ receptor availability of the right insula in a positron emission tomography imaging study (Ballanger et al., 2012). All these evidence support the link between the right insular cortex with subjective feeling states and emotional self-awareness, which is relevant to depression (Farb et al., 2013). The novelty of the present study lies in the fact that we used the insular seed volume to depict the depression severity in patients with PD-MCI, supporting possible trajectory of alpha-synucleinopathy-related neurodegenerative processes in emotional controls. Similar to our patients with PD-MCI-D, decreased frontoinsular GM volume was found in patients with major depressive disorder (Takahashi et al., 2010).

\section{SN and Depressive State in PD-MCI}

The structural covariance data might be considered as an indirect measurement of connectivity effectiveness. In addition to the insular seed, decreased structural association within the SN in our PD-MCI-D group provides a possible neural bridge linking key cortical hubs to depressive state in PD-MCI. Reduced BOLD between the anterior insula and ACC in patients with major depressive disorder has been reported (Veer et al., 2010). Stronger correlations may suggest greater regional connectivity and synchronized GM loss in the regions targeted by the 
TABLE 3 | Seed-based structural covariance network.

\begin{tabular}{|c|c|c|c|c|c|}
\hline & $x$ & $y$ & $z$ & Z-score & Voxels \\
\hline \multicolumn{6}{|l|}{ PD-MCI-ND } \\
\hline Right Insula & 36 & 18 & 4.5 & 39.1912 & 44934 \\
\hline Left inferior parietal & -31.5 & -73.5 & 40.5 & 7.0032 & 2060 \\
\hline Left middle cingulate cortex & -1.5 & -34.5 & 39 & 5.5926 & 369 \\
\hline Right middle cingulate cortex & 10.5 & 3 & 43.5 & 4.6547 & 127 \\
\hline Right inferior temporal & 48 & 3 & -36 & 4.6695 & 147 \\
\hline Right middle temporal & 52.5 & -64.5 & 3 & 7.0035 & 2524 \\
\hline Left superior temporal & -52.5 & -9 & 1.5 & 4.3635 & 179 \\
\hline Left inferior frontal & -42 & 6 & 25.5 & 4.7499 & 204 \\
\hline \multicolumn{6}{|l|}{ PD-MCl-D } \\
\hline Left medial orbitofrontal & -7.5 & 52.5 & -3 & 6.2846 & 2901 \\
\hline Right middle temporal & 57 & 4.5 & -22.5 & 6.468 & 126 \\
\hline Left inferior temporal & -60 & -25.5 & -19.5 & 6.1856 & 266 \\
\hline Left hippocampus & -15 & -9 & -15 & 5.37 & 152 \\
\hline Right insula & 36 & 18 & 6 & 23.5801 & 3886 \\
\hline Right hippocampus & 37.5 & -21 & -16.5 & 4.232 & 105 \\
\hline Left insula & -37.5 & 9 & 3 & 9.1382 & 2164 \\
\hline Right anterior cingulate cortex & 9 & 39 & 18 & 4.8467 & 114 \\
\hline Left precuneus & -10.5 & -54 & 34.5 & 6.1958 & 1100 \\
\hline Right middle cingulate cortex & 6 & 22.5 & 31.5 & 4.8313 & 496 \\
\hline Left middle cingulate cortex & -6 & -22.5 & 37.5 & 5.2058 & 136 \\
\hline
\end{tabular}

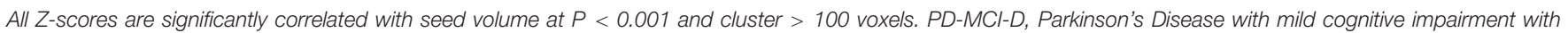
depression; PD-MCI-ND, PD-MCI without depression.

pathological process. The findings of the PD-MCI-D group may reflect segregated and less integrated components, compared with PD-MCI-ND group in the SN that could predict depressive scores. The peak clusters showing differences in covariance strength between the two PD-MCI groups in this study included the left lingual gyrus, the right hippocampal and the left caudate.

\section{Role of Lingual Gyrus Region and Depression}

Our result showed a clear association between the right insular seed volume and depressive severity. However, the pattern of volumetric correlation was not restricted to the insula. The cluster volume of the lingual gyrus also showed an inverse correlation with the GDS, with worse depressive scores being specifically associated with smaller cluster volume. How might larger cluster volume in the lingual gyrus contribute to better performance of depressive scores? One possibility is that individuals with larger lingual gyrus cluster volume are able to process happy faces normally. This mechanism might be supported by the functional imaging studies in patients with depression that revealed abnormal processing of happy faces in the lingual gyrus in patients with depression (Fu et al., 2007). Another possibility is that larger cluster lingual gyrus volume would have more abundant 5-HT2 receptors, supporting better neurochemical stimulation for a less depressive mood. A previous study has found that diminished 5-HT2 receptors in the lingual gyrus are correlated with depressive symptoms (Yatham et al., 2010). The lingual gyrus plays an important role in the connection between the visual pathway and limbic system (Conrad and Stumpf, 1975).
Besides, a larger volume of lingual gyrus can be a predictor of early antidepressant response in patients with major depressive disorder (Jung et al., 2014). These studies suggest that functional and structural abnormality in the lingual gyrus can be a predictor of depressive severity. Taken together, our data for the first time demonstrated that both the insula and lingual seed volume were associated with depressive severity. Therefore, our finding suggests the link between the insula and lingual gyrus within the $\mathrm{SN}$ might be a potential network basis for depression in PD-MCI.

\section{Role of Other Peak Cluster - the Right Hippocampus and Left Caudate}

In PD, correlations between MMSE and the density of Lewy neurites in the hippocampus and amygdala were reported (Churchyard and Lees, 1997). In this study, significant relationships between the right hippocampal volume with the generation of fruit category and the left caudate volume with VOSP were found. The finding of the relationship between fruit verbal fluency with the hippocampus has received attention before (Gleissner and Elger, 2001), and notably, is concordant with a recent study where the hippocampus was implicated in the late phase of semantic verbal fluency (Catheline et al., 2015). As our patients with PD-MCI did not perform worse than the NC on the verbal fluency test in the fruit category, the better scores are possibly dependent on the hippocampal network integrity, in contrast to the fact that early speech production is dependent on executive networks. The decreased dopamine transporter in the caudate has been reported to confer impairment of visuospatial skills (Marquie et al., 2014). 


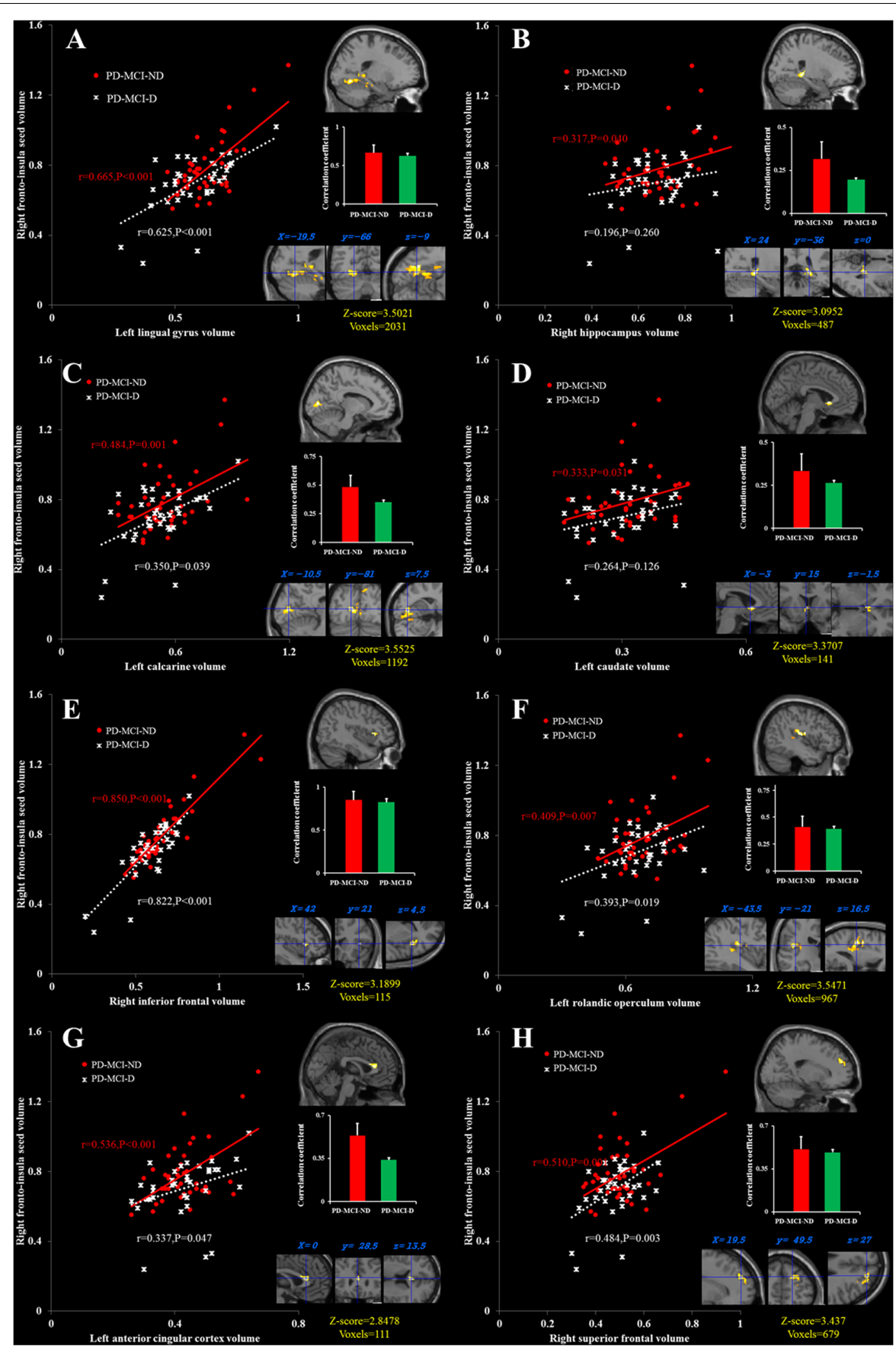

FIGURE 3 | Peak voxels that expressed decreased structural association in the PD-MCI-D group compared with the PD-MCl-ND group, and correlations between the gray matter volumes extracted from a 4-mm radius sphere centered on the frontoinsular seed and a 4-mm radius sphere centered on these peak voxels, including the left lingual gyrus (A), the right hippocampus (B), the left calcarine (C), the left caudate (D), the right inferior frontal cortex (E), the left rolandic operculum $\mathbf{( F )}$, the left anterior cingulate cortex, (G) and the right superior frontal cortex $\mathbf{( H )}$. Red dots represent PD-MCI-ND, and white crosses represent PD-MCI-D.

\section{Atrophic Patterns in PD-MCI}

In VBM analysis, we found significant GM alterations in the bilateral frontotemporal and bilateral occipital regions in the PDMCI group. The involvement of the frontotemporal cortex in PD-MCI is supported by a recent study that is related to the prediction of cognitive function (Xu et al., 2016). The significant decrease in the volume of right cerebellum in patients with PDMCI is consistent with the literature observation that the lower GM volume in the cerebellum contributes to gait difficulty in Parkinson's disease (Rosenberg-Katz et al., 2013). Larger GM reduction in the $\mathrm{PD}-\mathrm{MCI}-\mathrm{ND}$ group was observed in the right fusiform, right hippocampus, and bilateral superior parietal gyrus 


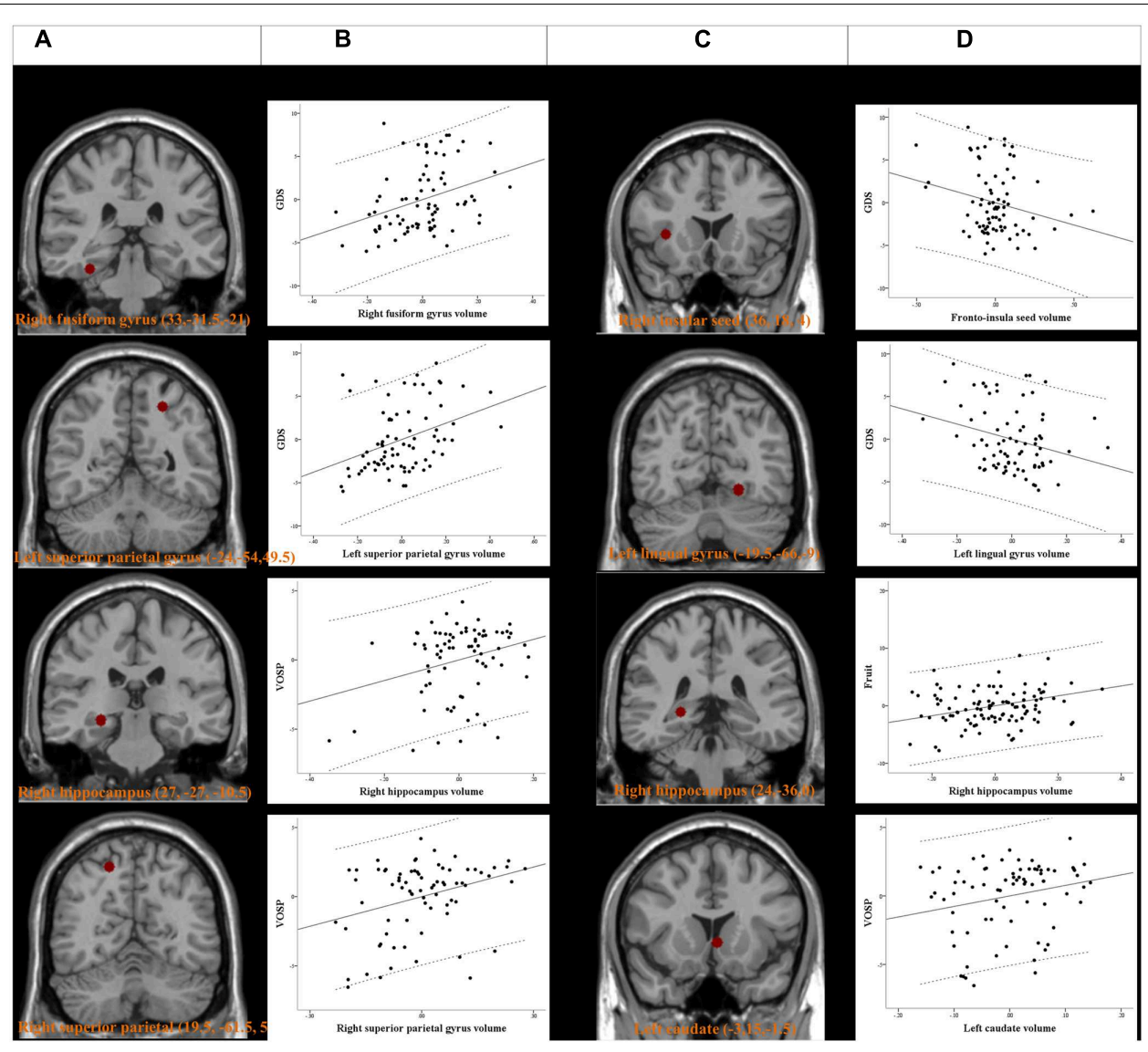

FIGURE 4 | Peak voxels of significant difference in the gray matter volume between PD-MCl-ND and PD-MCI with depression (PD-MCI-D) (A). Peak voxels that expressed decreased structural association in the PD-MCl-D group compared with the PD-MCl-ND group (C). The seed or voxels are significantly correlated with neurobehavioral scores. (B,D) Partial regression plots with 95\% confidence interval (dashed line) showing the relationships among the neurobehavioral scores, fronto-insula seed volume, and volume of peak clusters with statistical significance. GDS, Geriatric Depression Scales; VOSP, Visual Object and Space Perception Battery. Residuals are plotted for each variable to adjust for the effects of age, gender, and education.

compared with the PD-MCI-D group. Studies have found that regional volume changes appear to be dynamic throughout the course of illness, with the structures being enlarged in the first period and reduced as the illness progresses. In previous studies using MRI findings, it has been showed that patients with major depressive disorder had increased GM volume in the posterior cingulate cortex, inferior frontal gyrus, and amygdala (Lorenzetti et al., 2009; Yang et al., 2015). The increased volume of the inferior frontal gyrus in major depressive disorder is positively correlated with sustained attention (Yang et al., 2015). As depression severity was relatively mild in our patients with PD-MCI, our results might suggest that the initial enlargement of the right fusiform gyrus, right hippocampus, and bilateral superior parietal gyri in the early phase of PD-MCI-D. The volume of the right hippocampus and superior parietal gyrus are positively correlated with the sustained visuospatial function of VOSP.

\section{Limitation}

Some limitations of our study need to be addressed. First, this is a cross-sectional investigation; longitudinal follow-up is needed to observe the temporal relationship between structural association and clinical symptoms and whether the observed differences reflect transient or long-term changes, as well as the effect of larger regional volume in PD-MCI-D on longitudinal cognitive change. Second, our study relies on clinical, rather than autopsy-proven diagnosis. However, our diagnosis follows standard diagnostic criteria that have a good diagnosis accuracy based on a clinicopathological study (Hughes et al., 1992). Third, in this study, we used seed-based analysis with an emphasis on SN. Using independent component analysis or resting state function MRI data in the future may further elucidate other networks involving depression in PD-MCI. It is important to note that only very specific set of regions, selected on the basis of their demonstrating an abnormal structural association relationship, were used to evaluate correlation with our neuropsychological assessments. In other words, in the absence of concurrent structural abnormality, our clinical-pathological relation is not a retest of previous work investigating relationships among fruit verbal fluency, visuospatial function, and regional GM atrophy. 


\section{CONCLUSION}

Overall, this study is the first to present a picture of the structural covariance of SN in patients with PD-MCI-D and PD-MCIND. The results of this study suggest that the frontoinsular seed volume within the $\mathrm{SN}$ influences the presence and severity of depression in patients with PD-MCI. The SN might be one of the possible mechanisms underlying the neural basis of depression in patients with PD-MCI while there is strength gradient between the seed and peak clusters from non-depressive to depressive PDMCI, thus supporting the importance of SN network alterations in PD degenerative network.

\section{ETHICS STATEMENT}

The study was approved by Chang Gung Memorial Hospital's Institutional Review Committee on Human Research (1051374C, 201601682B0).

\section{REFERENCES}

Aarsland, D., Pahlhagen, S., Ballard, C. G., Ehrt, U., and Svenningsson, P. (2012). Depression in Parkinson disease-epidemiology, mechanisms and management. Nat. Rev. Neurol. 8, 35-47. doi: 10.1038/nrneurol.2011.189

Alexander-Bloch, A., Giedd, J. N., and Bullmore, E. (2013). Imaging structural co-variance between human brain regions. Nat. Rev. Neurosci. 14, 322-336. doi: $10.1038 / \mathrm{nrn} 3465$

Amieva, H., Lafont, S., Rouch-Leroyer, I., Rainville, C., Dartigues, J. F., Orgogozo, J. M., et al. (2004). Evidencing inhibitory deficits in Alzheimer's disease through interference effects and shifting disabilities in the Stroop test. Arch. Clin. Neuropsychol. 19, 791-803. doi: 10.1016/j.acn.2003.09.006

Aybek, S., Lazeyras, F., Gronchi-Perrin, A., Burkhard, P. R., Villemure, J. G., and Vingerhoets, F. J. (2009). Hippocampal atrophy predicts conversion to dementia after STN-DBS in Parkinson's disease. Parkinsonism Relat. Disord. 15, 521-524. doi: 10.1016/j.parkreldis.2009.01.003

Ballanger, B., Klinger, H., Eche, J., Lerond, J., Vallet, A. E., Le Bars, D., et al. (2012). Role of serotonergic $1 \mathrm{~A}$ receptor dysfunction in depression associated with Parkinson's disease. Mov. Disord. 27, 84-89. doi: 10.1002/mds.23895

Beyer, M. K., Janvin, C. C., Larsen, J. P., and Aarsland, D. (2007). A magnetic resonance imaging study of patients with Parkinson's disease with mild cognitive impairment and dementia using voxel-based morphometry. J. Neurol. Neurosurg. Psychiatry 78, 254-259. doi: 10.1136/jnnp.2006.093849

Braak, H., Bohl, J. R., Muller, C. M., Rub, U., de Vos, R. A., and Del Tredici, K. (2006). Stanley Fahn Lecture 2005: the staging procedure for the inclusion body pathology associated with sporadic Parkinson's disease reconsidered. Mov. Disord. 21, 2042-2051. doi: 10.1002/mds.21065

Bressler, S. L., and Menon, V. (2010). Large-scale brain networks in cognition: emerging methods and principles. Trends Cogn. Sci. 14, 277-290. doi: 10.1016/ j.tics.2010.04.004

Brown, R., and Jahanshahi, M. (1995). Depression in Parkinson's disease: a psychosocial viewpoint. Adv. Neurol. 65, 61-84.

Burn, D. J. (2002). Beyond the iron mask: towards better recognition and treatment of depression associated with Parkinson's disease. Mov. Disord. 17, 445-454. doi: $10.1002 / \mathrm{mds} .10114$

Cardoso, E. F., Maia, F. M., Fregni, F., Myczkowski, M. L., Melo, L. M., Sato, J. R., et al. (2009). Depression in Parkinson's disease: convergence from voxelbased morphometry and functional magnetic resonance imaging in the limbic thalamus. Neuroimage 47, 467-472. doi: 10.1016/j.neuroimage.2009.04.059

Catheline, G., Amieva, H., Dilharreguy, B., Bernard, C., Duperron, M. G., Helmer, C., et al. (2015). Semantic retrieval over time in the aging brain: structural evidence of hippocampal contribution. Hippocampus 25, 1008-1016. doi: 10.1002/hipo. 22423

\section{AUTHOR CONTRIBUTIONS}

Research project conception, organization, execution: Y-TC, C-CC, C-WH, W-NC, M-KW, and S-WH. Statistical analysis execution and review and critique: J-JL and C-YL. Manuscript preparation: Y-TC, C-HL, and C-CC.

\section{FUNDING}

This study was supported by grants from the Chang Gung Memorial Hospital (CMRPG8E0751).

\section{ACKNOWLEDGMENTS}

The authors wish to thank Dr. Yen-Yu Chen for statistical analysis suggestion.

Chang, C. C., Chang, Y. Y., Chang, W. N., Lee, Y. C., Wang, Y. L., Lui, C. C., et al. (2009). Cognitive deficits in multiple system atrophy correlate with frontal atrophy and disease duration. Eur. J. Neurol. 16, 1144-1150. doi: 10.1111/j. 1468-1331.2009.02661.x

Chang, C. C., Hsu, J. L., Chang, W. N., Huang, S. H., Huang, C. W., Chang, Y. T., et al. (2016). Metabolic covariant network in relation to nigrostriatal degeneration in carbon monoxide intoxication-related parkinsonism. Front. Neurosci. 10:187. doi: 10.3389/fnins.2016.00187

Chang, C. C., Liu, J. S., Chang, Y. Y., Chang, W. N., Chen, S. S., and Lee, C. H. (2008). (99m)Tc-ethyl cysteinate dimer brain SPECT findings in early stage of dementia with Lewy bodies and Parkinson's disease patients: a correlation with neuropsychological tests. Eur. J. Neurol. 15, 61-65.

Churchyard, A., and Lees, A. J. (1997). The relationship between dementia and direct involvement of the hippocampus and amygdala in Parkinson's disease. Neurology 49, 1570-1576. doi: 10.1212/WNL.49.6.1570

Clos, M., Rottschy, C., Laird, A. R., Fox, P. T., and Eickhoff, S. B. (2014). Comparison of structural covariance with functional connectivity approaches exemplified by an investigation of the left anterior insula. Neuroimage 99, 269-280. doi: 10.1016/j.neuroimage.2014.05.030

Conrad, C. D., and Stumpf, W. E. (1975). Direct visual input to the limbic system: crossed retinal projections to the nucleus anterodorsalis thalami in the tree shrew. Exp. Brain Res. 23, 141-149. doi: 10.1007/BF00235456

Domellof, M. E., Ekman, U., Forsgren, L., and Elgh, E. (2015). Cognitive function in the early phase of Parkinson's disease, a five-year follow-up. Acta Neurol. Scand. 132, 79-88. doi: 10.1111/ane.12375

Farb, N. A., Segal, Z. V., and Anderson, A. K. (2013). Attentional modulation of primary interoceptive and exteroceptive cortices. Cereb. Cortex 23, 114-126. doi: 10.1093/cercor/bhr385

Fu, C. H., Williams, S. C., Brammer, M. J., Suckling, J., Kim, J., Cleare, A. J., et al. (2007). Neural responses to happy facial expressions in major depression following antidepressant treatment. Am. J. Psychiatry 164, 599-607. doi: 10.1176/ajp.2007.164.4.599

Gleissner, U., and Elger, C. E. (2001). The hippocampal contribution to verbal fluency in patients with temporal lobe epilepsy. Cortex 37, 55-63. doi: 10.1016/ S0010-9452(08)70557-4

Huang, C. W., Tsai, M. H., Chen, N. C., Chen, W. H., Lu, Y. T., Lui, C. C., et al. (2015). Clinical significance of circulating vascular cell adhesion molecule-1 to white matter disintegrity in Alzheimer's dementia. Thromb. Haemost. 114, 1230-1240. doi: 10.1160/TH14-11-0938

Hughes, A. J., Ben-Shlomo, Y., Daniel, S. E., and Lees, A. J. (1992). What features improve the accuracy of clinical diagnosis in Parkinson's disease: a clinicopathologic study. Neurology 42, 1142-1146. doi: 10.1212/WNL.42. 6.1142 
Jabbi, M., and Keysers, C. (2008). Inferior frontal gyrus activity triggers anterior insula response to emotional facial expressions. Emotion 8, 775-780. doi: 10.1037/a0014194

Janvin, C. C., Larsen, J. P., Aarsland, D., and Hugdahl, K. (2006). Subtypes of mild cognitive impairment in Parkinson's disease: progression to dementia. Mov. Disord. 21, 1343-1349. doi: 10.1002/mds.20974

Jokinen, P., Brück, A., Aalto, S., Forsback, S., Parkkola, R., and Rinne, J. O. (2009). Impaired cognitive performance in Parkinson's disease is related to caudate dopaminergic hypofunction and hippocampal atrophy. Parkinsonism Relat. Disord. 15, 88-93. doi: 10.1016/j.parkreldis.2008.03.005

Jung, J., Kang, J., Won, E., Nam, K., Lee, M. S., Tae, W. S., et al. (2014). Impact of lingual gyrus volume on antidepressant response and neurocognitive functions in Major Depressive Disorder: a voxel-based morphometry study. J. Affect. Disord. 169, 179-187. doi: 10.1016/j.jad.2014.08.018

Kostic, V. S., Agosta, F., Petrovic, I., Galantucci, S., Spica, V., JecmenicaLukic, M., et al. (2010). Regional patterns of brain tissue loss associated with depression in Parkinson disease. Neurology 75, 857-863. doi: 10.1212/WNL. 0b013e3181f11c1d

Lin, P. H., Tsai, S. J., Huang, C. W., Mu-En, L., Hsu, S. W., Lee, C. C., et al. (2016). Dose-dependent genotype effects of BDNF Val66Met polymorphism on default mode network in early stage Alzheimer's disease. Oncotarget 7, 54200-54214. doi: 10.18632 /oncotarget.11027

Litvan, I., Goldman, J. G., Troster, A. I., Schmand, B. A., Weintraub, D., Petersen, R. C., et al. (2012). Diagnostic criteria for mild cognitive impairment in Parkinson's disease: Movement Disorder Society Task Force guidelines. Mov Disord. 27, 349-356. doi: 10.1002/mds.24893

Lorenzetti, V., Allen, N. B., Fornito, A., and Yucel, M. (2009). Structural brain abnormalities in major depressive disorder: a selective review of recent MRI studies. J. Affect. Disord. 117, 1-17. doi: 10.1016/j.jad.2008.11.021

Lu, P. H., Edland, S. D., Teng, E., Tingus, K., Petersen, R. C., and Cummings, J. L. (2009). Donepezil delays progression to $A D$ in MCI subjects with depressive symptoms. Neurology 72, 2115-2121. doi: 10.1212/WNL.0b013e3181aa52d3

Marc, L. G., Raue, P. J., and Bruce, M. L. (2008). Screening performance of the 15item geriatric depression scale in a diverse elderly home care population. Am. J. Geriatr. Psychiatry 16, 914-921. doi: 10.1097/JGP.0b013e318186bd67

Marquie, M., Locascio, J. J., Rentz, D. M., Becker, J. A., Hedden, T., Johnson, K. A., et al. (2014). Striatal and extrastriatal dopamine transporter levels relate to cognition in Lewy body diseases: an (11)C altropane positron emission tomography study. Alzheimers Res. Ther. 6:52. doi: 10.1186/s13195-014-0052-7

Martin, W. R., Wieler, M., Gee, M., and Camicioli, R. (2009). Temporal lobe changes in early, untreated Parkinson's disease. Mov. Disord. 24, 1949-1954. doi: $10.1002 / \mathrm{mds} .22680$

Mechelli, A., Crinion, J. T., Noppeney, U., O’Doherty, J., Ashburner, J., Frackowiak, R. S., et al. (2004). Neurolinguistics: structural plasticity in the bilingual brain. Nature 431:757. doi: 10.1038/431757a

Menon, V. (2015). "Salience network," in Brain Mapping: An Encyclopedic Reference, Vol. 2, ed. A. W. Toga (Cambridge, MA: Academic Press), 597-611. doi: 10.1016/B978-0-12-397025-1.00052-X

Modrego, P. J., and Ferrandez, J. (2004). Depression in patients with mild cognitive impairment increases the risk of developing dementia of Alzheimer type: a prospective cohort study. Arch. Neurol. 61, 1290-1293. doi: 10.1001/archneur. 61.8 .1290

Montembeault, M., Rouleau, I., Provost, J. S., and Brambati, S. M. (2016). Altered gray matter structural covariance networks in early stages of Alzheimer's disease. Cereb. Cortex 26, 2650-2662. doi: 10.1093/cercor/bhv105

Rapport, L. J., Millis, S. R., and Bonello, P. J. (1998). Validation of the Warrington theory of visual processing and the visual object and space perception battery. J. Clin. Exp. Neuropsychol. 20, 211-220. doi: 10.1076/jcen.20.2.211.1169

Reginold, W., Duff-Canning, S., Meaney, C., Armstrong, M. J., Fox, S., Rothberg, B., et al. (2013). Impact of mild cognitive impairment on healthrelated quality of life in Parkinson's disease. Dement. Geriatr. Cogn. Disord. 36, 67-75. doi: 10.1159/000350032
Reitan, R. M. (1955). The relation of the trail making test to organic brain damage. J. Consult. Psychol. 19, 393-394. doi: 10.1037/h0044509

Remy, P., Doder, M., Lees, A., Turjanski, N., and Brooks, D. (2005). Depression in Parkinson's disease: loss of dopamine and noradrenaline innervation in the limbic system. Brain 128, 1314-1322. doi: 10.1093/brain/awh445

Rosenberg-Katz, K., Herman, T., Jacob, Y., Giladi, N., Hendler, T., and Hausdorff, J. M. (2013). Gray matter atrophy distinguishes between Parkinson disease motor subtypes. Neurology 80, 1476-1484. doi: 10.1212/WNL. 0b013e31828cfaa 4

Seeley, W. W., Crawford, R. K., Zhou, J., Miller, B. L., and Greicius, M. D. (2009). Neurodegenerative diseases target large-scale human brain networks. Neuron 62, 42-52. doi: 10.1016/j.neuron.2009.03.024

Seeley, W. W., Menon, V., Schatzberg, A. F., Keller, J., Glover, G. H., Kenna, H., et al. (2007). Dissociable intrinsic connectivity networks for salience processing and executive control. J. Neurosci. 27, 2349-2356. doi: 10.1523/JNEUROSCI. 5587-06.2007

Song, S. K., Lee, J. E., Park, H. J., Sohn, Y. H., Lee, J. D., and Lee, P. H. (2011). The pattern of cortical atrophy in patients with Parkinson's disease according to cognitive status. Mov. Disord. 26, 289-296. doi: 10.1002/mds.23477

Spreen, O., and Strauss, E. (1998). "Geriatric depression scale (GDS)," in A Compendium of Neuropsychological Tests, eds O. Spreen and E. Strauss (New York, NY: Oxford University Press), 612-616.

Takahashi, T., Yucel, M., Lorenzetti, V., Tanino, R., Whittle, S., Suzuki, M., et al. (2010). Volumetric MRI study of the insular cortex in individuals with current and past major depression. J. Affect. Disord. 121, 231-238. doi: 10.1016/j.jad. 2009.06.003

Veer, I. M., Beckmann, C. F., van Tol, M. J., Ferrarini, L., Milles, J., Veltman, D. J., et al. (2010). Whole brain resting-state analysis reveals decreased functional connectivity in major depression. Front. Syst. Neurosci. 4:41. doi: 10.3389/fnsys. 2010.00041

Xu, Y., Yang, J., Hu, X., and Shang, H. (2016). Voxel-based meta-analysis of gray matter volume reductions associated with cognitive impairment in Parkinson's disease. J. Neurol. 263, 1178-1187. doi: 10.1007/s00415-016-8122-3

Yang, X., Ma, X., Huang, B., Sun, G., Zhao, L., Lin, D., et al. (2015). Gray matter volume abnormalities were associated with sustained attention in unmedicated major depression. Compr. Psychiatry 63, 71-79. doi: 10.1016/j.comppsych.2015. 09.003

Yatham, L. N., Liddle, P. F., Lam, R. W., Zis, A. P., Stoessl, A. J., Sossi, V. et al. (2010). Effect of electroconvulsive therapy on brain 5-HT(2) receptors in major depression. Br. J. Psychiatry 196, 474-479. doi: 10.1192/bjp.bp.109. 069567

Zaki, J., Ochsner, K. N., Hanelin, J., Wager, T. D., and Mackey, S. C. (2007). Different circuits for different pain: patterns of functional connectivity reveal distinct networks for processing pain in self and others. Soc. Neurosci. 2, 276-291. doi: 10.1080/17470910701401973

Zimmerman, M., Emmert-Aronson, B. O., and Brown, T. A. (2011). Concordance between a simpler definition of major depressive disorder and diagnostic and statistical manual of mental disorders, fourth edition: an independent replication in an outpatient sample. Compr. Psychiatry 52, 261-264. doi: 10.1016/j.comppsych.2010.07.009

Conflict of Interest Statement: The authors declare that the research was conducted in the absence of any commercial or financial relationships that could be construed as a potential conflict of interest.

Copyright (๑ 2018 Chang, Lu, Wu, Hsu, Huang, Chang, Lien, Lee and Chang. This is an open-access article distributed under the terms of the Creative Commons Attribution License (CC BY). The use, distribution or reproduction in other forums is permitted, provided the original author(s) or licensor are credited and that the original publication in this journal is cited, in accordance with accepted academic practice. No use, distribution or reproduction is permitted which does not comply with these terms. 